\section{Erysipelothrix rhusiopathiae bacteremia without endocarditis: rapid identification from positive blood culture by MALDI-TOF mass spectrometry. A case report and literature review}

\section{Luigi Principe, Silvia Bracco, Carola Mauri, Silvia Tonolo, Beatrice Pini, Francesco Luzzaro}

Microbiology and Virology Unit, A. Manzoni Hospital, Lecco, Italy

\section{Abstract}

Erysipelothrix rhusiopathiae is a Gram-positive bacillus that is infrequently responsible for infections in humans. Three forms have been classified: a localized cutaneous form (erysipeloid) caused by traumatic penetration of $E$. rhusiopathiae, a generalized cutaneous form and a septicemic form. The latter type of disease has been previously associated with a high incidence of endocarditis. Here we report a case of $E$. rhusiopathiae bacteremia in a 74year-old man, probably started from an erysipeloid form, in which endocarditis did not develop. This case presents some particular and uncommon features: i) no correlation with animal source; ii) correlation between bacteremia and erysipeloid lesion; iii) absence of endocarditis. MALDI-TOF mass spectrometry allowed to obtain a rapid identification (within 4 hours from bottle positivity) of $E$. rhusiopathiae. Together with direct antimicrobial susceptibility testing, this approach could improve the rate of appropriate therapy for bloodstream infections due to this fastidious pathogen.

\section{Introduction}

Erysipelothrix rhusiopathiae is a Gram-positive bacillus that is infrequently responsible for infections in humans. Human disease, although rare, can originate from animal or environmental sources. To this regard, animal sources are often associated with occupational exposure (butchers, fishermen, fish handlers, veterinarians), with pigs as the most important reservoir. ${ }^{1}$ On the other hand, soil, food scraps and water contaminated by infected animals represent the most common environmental sources. The organism is widespread and occurs in decomposing organic matter. Notably, E. rhusiopathiae can survive in soil for several weeks. ${ }^{2}$ Human disease is classified into three forms: a localized cutaneous form (erysipeloid) caused by traumatic penetration of $E$. rhusiopathiae, a generalized cutaneous form and a septicemic form. The latter type of disease has been previously associated with a high incidence of endocarditis. In a previous study concerning invasive infection cases since 1912 , it was reported that about $90 \%$ of cases of $E$. rhusiopathiae bacteremia result in endocarditis. ${ }^{3}$ This association has been recently questioned, since some cases of $E$. rhusiopathiae bacteremia without subsequent endocarditis have been reported in the most recent literature. ${ }^{4-8}$ Here we report a case of $E$. rhusiopathiae bacteremia, probably started from an erysipeloid form, in which endocarditis did not develop. Furthermore, we used the PubMed Database to search for recent case reports of bloodstream infections caused by $E$. rhusiopathiae.

\section{Case Report}

An Italian citizen (male, 74 years old) presented to the emergency department of the San Leopoldo Mandic Hospital in Merate (Lecco, Italy) on August, 2014. The patient was in treatment with oral anticoagulant therapy due to cardiopathy and cerebral vasculopathy. On admission, he had a low grade fever $\left(38.4^{\circ} \mathrm{C}\right)$, hearth rate 100 beats per minute, blood pressure $125 / 75 \mathrm{~mm}$ of $\mathrm{Hg}$, white blood cell count $4.2 \times 10^{9} / \mathrm{L}$. In addition, he showed difficulty in his movement due to a polymorphic erythema on the right leg characterized by well-defined and raised borders with a localized edematous skin portion and reddening. Based on laboratory and clinical data (modified early warning score $=4$ ), the presence of sepsis was suspected. ${ }^{9}$ Together with other interventions (as appropriate for sepsis condition), two blood cultures were performed and sent to the laboratory where they were promptly incubated in the BacT/ALERT instrument (bioMérieux, Marcy l'Etoile, France). No biological samples from leg were sent to the microbiology laboratory. Therapy with ceftriaxone ( $1 \mathrm{~g}$ twice daily) plus azithromycin (500 mg daily) was then initiated. The patient recalled that the lesion, then classified as erysipelas, had been present for about two months. No contact with animals were reported. However, physicians observed that the patient lived in non-optimal hygienic conditions. After a $48 \mathrm{~h}$ incubation period, an aerobic bottle from blood cultures performed at the emergency department was flagged positive by the BacT/ALERT instrument (bioMérieux). Direct microscopic examination based on Gram staining evidenced the presence of Gram-variable rods. Bacterial identification
Correspondence: Francesco Luzzaro, Microbiology and Virology Unit, A. Manzoni Hospital, Via dell'Eremo 9/11, 23900 Lecco, Italy. Tel.: +39.0341.489630 - Fax: +39.0341 .489601 .

E-mail: f.luzzaro@asst-lecco.it

Key words: Erysipelas; skin and soft tissue infections; antimicrobial treatment; empirical therapy.

Contributions: LP performed laboratory diagnosis and drafted the manuscript; $\mathrm{SB}, \mathrm{CM}$ and $\mathrm{BP}$ performed susceptibility tests, analysed data and contributed to laboratory diagnosis; ST provided clinical information about the patient; FL conceived of the study, participated in its design and coordination, and critically revised the draft manuscript.

Conflict of interest: the authors declare no potential conflict of interest.

Received for publication: 17 December 2015.

Revision received: 25 January 2016.

Accepted for publication: 25 January 2016.

This work is licensed under a Creative Commons Attribution-NonCommercial 4.0 International License (CC BY-NC 4.0).

(C) Copyright L. Principe et al., 2016

Licensee PAGEPress, Italy

Infectious Disease Reports 2016; 8:6368

doi:10.4081/idr.2016.6368

was performed directly from positive blood culture bottle by matrix-assisted laser desorption ionization time-of-flight (MALDI-TOF) mass spectrometry (VITEK MS, bioMérieux) using the following procedure. An aliquot $(2.5 \mathrm{~mL})$ of the blood culture was transferred in a tube with gel separator (BD Vacutainer® Blood Collection Tubes, Becton, Dickinson and Company, Milan, Italy) and centrifuged at 3500 rpm for 10 minutes. The supernatant was discarded and the pellet was inoculated on two blood agar plates incubated at $36^{\circ} \mathrm{C}$ in $\mathrm{O}_{2}$ and $5 \% \mathrm{CO}_{2}$, respectively. After a short term incubation period (4 hours), microbial identification was obtained by the VITEK MS directly from bacterial growth on agar plates. Microorganisms recovered from agar plates were directly applied to VITEK MS target slide in duplicate (two spots for each isolate) and were covered with one microliter of CHCA ( $\alpha$ Cyano-4-hydroxycinnamic acid) matrix.

E. rhusiopathiae was identified according to the observation of Gram-variable rods as obtained by Gram staining. Next to rapid identification with VITEK MS, a suspension, adjusted to a 0.5 McFarland turbidity standard, was created directly from bacterial growth and used for direct antimicrobial susceptibility testing by Etest strips (bioMérieux) using the Mueller-Hinton Fastidious agar (bioMérieux). 
The minimum inhibitory concentrations of 7 antibiotics were evaluated, including penicillin G, cefotaxime, imipenem, ciprofloxacin, levofloxacin, clindamycin, and vancomycin. Since no specific criteria were available for $E$. rhusiopathiae, results were arbitrarily interpreted according to EUCAST criteria and non-species related breakpoints. ${ }^{10}$

As shown in Table 1, antimicrobial agents used for empirical treatment resulted highly active against the etiological agent isolated from blood. A transesophageal echocardiography did not reveal valve vegetation. Ten days after admission, the patient was discharged without fever and in good conditions. The follow-up conducted seven months after discharge showed good clinical conditions (taking into account his underlying illness). The erysipelas on the right leg was completely cured.

\section{Discussion and Conclusions}

This report describes a case of $E$. rhusiopathiae bacteremia without endocarditis in an immunocompetent patient, successfully treated with ceftriaxone plus azithromycin, presumably started from a localized skin form.

Similar cases are overall uncommon. Table 2 summarizes clinical features of $E$. rhusiopathiae invasive case reports, searched in the most recent literature using the PubMed Database (only articles in English have been taken into account). ${ }^{3-35}$

With respect to previous reports, our case presents some particular and uncommon features. First, erysipelas is most often caused by Streptococcus pyogenes or Staphylococcus aureus, less so by group B, C or G streptococci. ${ }^{11}$ For this reason, Erysipelothrix infections are considered uncommon and especially nonoccupational cases are very rare. In our case the patient lived in non-optimal hygienic conditions but he had not been in contact with any animal, so the source of infection could be the soil contaminated by the microorganism and the infection could occur by traumatic penetration in the skin through tiny breaks. Overall, a total of 32 cases (including our case) have been recently reported. Of importance, our search revealed only two other cases associated to poor hygienic conditions (in addition to our case), while an exposure to animal sources was mostly present (Table 2).

Second, bacteremic infection, with or without endocarditis, was previously considered most commonly a primary infection rather than dissemination from localized cutaneous lesion. ${ }^{1,5,12}$ In contrast to this assumption, the recent literature shows an increasing rate of bacteremic infections started from localized cutaneous lesion (Table 2). Particularly, in our case, bacteremia developed subsequently an erysipeloid lesion.

Third, E. rhusiopathiae bacteremia is most commonly associated with severe clinical illness and is often complicated with endocarditis. ${ }^{1}$ More than one half of patients with systemic infection had predisposing factors such alcohol or drug dependence, immunosuppression, and chronic liver disease (Table 2). Furthermore, our search showed that more than one third of the patients (34.4\%) with invasive $E$. rhusiopathiae infections developed endocarditis (Table 2). This rate is much lower than that reported by Gorby and Peacock, in which about $90 \%$ of cases of $E$. rhusiopathiae bacteremia result in endocarditis. ${ }^{3}$ However, in contrast to these evidences, in our case the patient did not present any of these clinical features and no involvement of the endocardium was detected.

It is noting that bacteremic $E$. rhusiopathiae infection may occur more commonly than reports suggest. It may be under-diagnosed and under-reported because the resemblance it bears to other infections and the problems that may be encountered in isolation and identification of this pathogen. Furthermore, Gram-positive rods cultured from blood are often dismissed as difteroids and not fully identified.

In our case, bacteremia without involvement of endocardium was resolved by treatment with ceftriaxone plus azithromycin. To this regard, penicillins and cephalosporins are the first line of choice for treating $E$. rhusiopathiae infections (see also Table 2) while this pathogen is naturally resistant to vancomycin, teicoplanin, daptomycin, gentamicin, netilmicin, polimyxin $\mathrm{B}$, tetracycline and trimethoprim/sulfamethoxazole. ${ }^{1}$ It is worth noting that some of the latter antimicrobial agents are commonly used as empirical therapy for treatment of suspected sepsis. Thus, early diagnosis of all forms of $E$. rhusiopathiae infection appears to be essential, especially in the case of endocarditis.
Of note, although $E$. rhusiopathiae is usually highly susceptible to penicillins and cephalosporins, all death patients described in Table 2 had been treated with such beta-lactam antibiotics. Based on data collected from the recent literature (Table 2), the overall mortality rate for bacteremia was $12.5 \%$. Three out of four deaths occurred in patients with endocarditis, whereas the remaining occurred in a patient with sepsis and oropharyngeal cancer. A previous study reported a mortality rate of $38 \%$ in patients with E. rhusiopathiae endocarditis. ${ }^{3}$ In our search the mortality rate for $E$. rhusiopathiae endocarditis accounted for $27.3 \%$ (Table 2). This rate may partly be explained by the use of vancomycin, often in combination with gentamicin, in the empirical therapy of endocarditis in patients who are allergic to penicillin and infected with Grampositive microorganisms. ${ }^{1}$ With respect to available alternative drugs, susceptibility data for quinolones are very rare in the literature.$^{4,6,19}$ In our case, we observed very low MIC values for ciprofloxacin and levofloxacin (Table 1), thus suggesting that these drugs could represent a valid therapeutic option, at least for patients with known allergy to betalactams, particularly if at risk of infectious endocarditis (i.e. patients with heart valve prosthesis).

The introduction of mass spectrometry in clinical microbiology laboratory has notably improved identification of microorganisms at the species level. To our best knowledge, this is the first report in which identification of $E$. rhusiopathiae has been performed directly from positive blood culture bottle by MALDITOF mass spectrometry. Identification by mass spectrometry and direct antimicrobial susceptibility testing performed on positive blood culture can provide results about 24 hours earlier than routine standard methods. This approach could allow to reduce the duration of empirical treatment and improve the rate of appropriate therapy for bloodstream infections caused by this fastidious pathogen.

Table 1. Susceptibility profile of Erysipelothrix rhusiopathiae blood isolate.

\begin{tabular}{lcc} 
Antimicrobial agent & MIC $(\mathrm{mg} / \mathrm{L})$ & Interpretation \\
Penicillin G & 0.004 & $\mathrm{~S}$ \\
Cefotaxime & 0.094 & $\mathrm{~S}$ \\
\hline Imipenem & 0.008 & $\mathrm{~S}$ \\
Ciprofloxacin & 0.094 & $\mathrm{~S}$ \\
\hline Levofloxacin & 0.064 & $\mathrm{~S}$ \\
Clindamycin & 0.094 & $\mathrm{~S}$ \\
\hline Vancomycin & 64 & -
\end{tabular}

Based on EUCAST criteria and non-species related breakpoints. ${ }^{10}$ No interpretation criteria are available for vancomycin. 


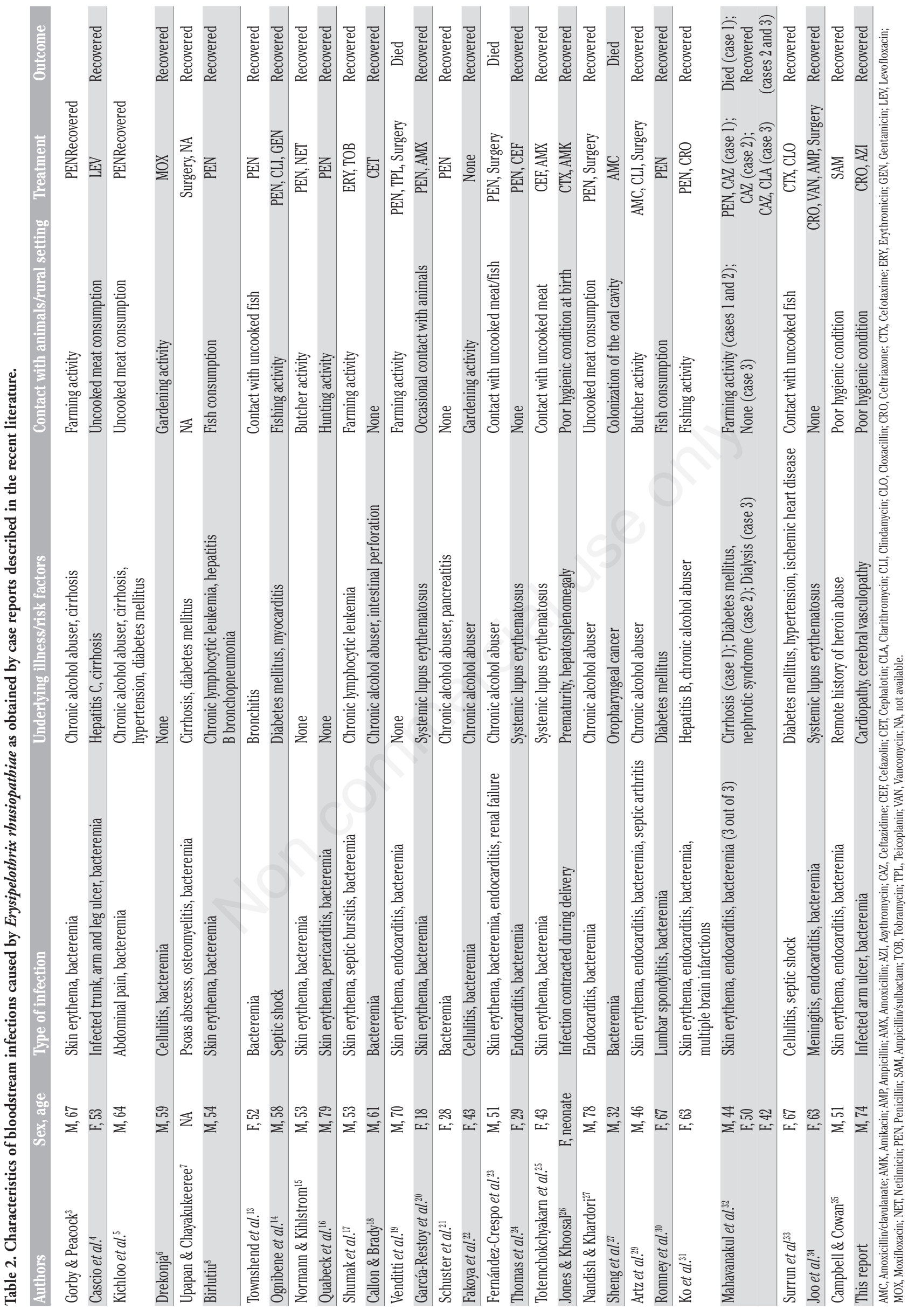




\section{References}

1. Wang Q, Chang BJ, Riley TV. Erysipelothrix rhusiopathiae. Vet Microbiol 2010;140:405-17.

2. Veraldi S, Girgenti V, Dassoni F, Gianotti R. Erysipeloid: a review. Clin Exp Dermatol 2009;34:859-62.

3. Gorby GL, Peacock JE Jr. Erysipelothrix rhusiopathiae endocarditis: microbiologic, epidemiologic, and clinical features of an occupational disease. Rev Infect Dis 1988;10:317-25.

4. Cascio A, Stassi G, Cacciola I, et al. Fever and rhomboid target lesion in decompensated cirrhosis. Lancet Infect Dis 2012;12:576

5. Kichloo AA, Hallac A, Mousavi B, Hirekhan 0. Nonspecific Erysipelothrix rhusiopathiae bacteremia in a patient with subclinical alcoholic liver disease. Case Rep Infect Dis 2013;2013:474593.

6. Drekonja DM. Erysipelothrix bacteremia without endocarditis: rare event or underreported occurrence? Diagn Microbiol Infect Dis 2013;77:280-1.

7. Upapan P, Chayakulkeeree M. Erysipelothrix rhusiopathiae bacteremia without endocarditis associated with psoas abscess: the first case report in Thailand. J Med Assoc Thai 2014;97:232-6.

8. Birlutiu V. Sepsis due to Erysipelothrix rhusiopathiae in a patient with chronic lymphocytic leukemia associated with bronchopneumonia due to Pseudomonas aeruginosa and Escherichia coli: a case report. Can J Infect Dis Med Microbiol 2015;26:108-10.

9. Burch VC, Tarr G, Morroni C. Modified early warning score predicts the need for hospital admission and inhospital mortality. Emerg Med J 2008;25:674-8.

10. European Committee on Antimicrobial Susceptibility Testing. Breakpoint tables for interpretation of MICs and zone diameters, version 4.0. Basel: EUCAST; 2014.

11. Bernard P. Management of common bacterial infections of the skin. Curr Opin Infect Dis 2008;21:122-8.

12. Feasi M, Bacigalupo L, Cappato S, et al.
Erysipelothrix rhusiopathiae intra-abdominal abscess. Int J Infect Dis 2010;14:81-3.

13. Townshend RH, Jephcott AE, Yekta MH. Erysipelothrix septicaemia without endocarditis. Br Med J 1973;1:464.

14. Ognibene FP, Cunnion RE, Gill V, et al. Erysipelothrix rhusiopathiae bacteremia presenting as septic shock. Am J Med 1985;78:861-4.

15. Normann B, Kihlström E. Erysipelothrix rhusiopathiae septicaemia. Scand J Infect Dis 1985;17:123-4.

16. Quabeck K, Müller J, Wendt F, Rosenthal E. Pericarditis in Erysipelothrix rhusiopathiae septicemia. Infection 1986;14:301.

17. Shumak SL, McDonald S, Baer P, Cowan DH. Erysipelothrix septicemia in an immunocompromised host. CMAJ 1987;136:273-4.

18. Callon RA Jr, Brady PG. Toothpick perforation of the sigmoid colon: an unusual case associated with Erysipelothrix rhusiopathiae septicemia. Gastrointest Endosc 1990;36:141-3.

19. Venditti M, Gelfusa V, Castelli F, et al. Erysipelothrix rhusiopathiae endocarditis. Eur J Clin Microbiol Infect Dis 1990;9:50-2.

20. García-Restoy E, Espejo E, Bella F, Llebot J. Bacteremia due to Erysipelothrix rhusiopathiae in immunocompromised hosts without endocarditis. Rev Infect Dis 1991;13:1252-3.

21. Schuster MG, Brennan PJ, Edelstein P. Persistent bacteremia with Erysipelothrix rhusiopathiae in a hospitalized patient. Clin Infect Dis 1993;17:783-4.

22. Fakoya A, Bendall RP, Churchill DR, et al. Erysipelothrix rhusiopathiae bacteraemia in a patient without endocarditis. J Infect 1995;30:180-1.

23. Fernández-Crespo P, Serra A, Bonet J, Gimenez M. Acute oliguric renal failure in a patient with an Erysipelothrix rhusiopathiae bacteremia and endocarditis. Nephron 1996;74:231.

24. Thomas N, Jesudason M, Mukundan U, et al. Infective endocarditis caused by Erysipelothrix rhusiopathiae in a patient with systemic lupus erythematosus. J Assoc Physicians India 1996;44:223.

25. Totemchokchyakarn K, Janwityanujit S,
Sathapatayavongs B, Puavilai S. Erysipelothrix rhusiopathiae septicemia in systemic lupus erythematosus. Int $\mathrm{J}$ Dermatol 1996;35:818-20.

26. Jones N, Khoosal M. Erysipelothrix rhusiopathiae septicemia in a neonate. Clin Infect Dis 1997;24:511.

27. Nandish S, Khardori N. Valvular and myocardial abscesses due to Erysipelothrix rhusiopathiae. Clin Infect Dis 1999;29:1351-2.

28. Sheng WH, Hsueh PR, Hung CC, et al. Fatal outcome of Erysipelothrix rhusiopathiae bacteremia in a patient with oropharyngeal cancer. J Formos Med Assoc 2000;99:431-4.

29. Artz AL, Szabo S, Zabel LT, Hoffmeister HM. Aortic valve endocarditis with paravalvular abscesses caused by Erysipelothrix rhusiopathiae. Eur J Clin Microbiol Infect Dis 2001;20:587-8.

30. Romney M, Cheung S, Montessori V. Erysipelothrix rhusiopathiae endocarditis and presumed osteomyelitis. Can J Infect Dis 2001;12:254-6.

31. Ko SB, Kim DE, Kwon HM, Roh JK. A case of multiple brain infarctions associated with Erysipelothrix rhusiopathiae endocarditis. Arch Neurol 2003;60:434-6.

32. Mahavanakul W, Limmathurotsakul D, Teerawattanasuk N, Peacock SJ. Invasive Erysipelothrix rhusiopathiae infection in northeast Thailand. Southeast Asian J Trop Med Public Health 2007;38:478-81.

33. Surrun SK, Jaufeerally FR, Sim HC. Erysipelothrix rhuseopathiae septicaemia with prolonged hypotension: a case report. Ann Acad Med Singapore 2008;37:251-2.

34. Joo EJ, Kang CI, Kim WS, et al.. Acute meningitis as an initial manifestation of Erysipelothrix rhusiopathiae endocarditis. J Infect Chemother 2011;17:703-5.

35. Campbell D, Cowan M. Septicemia and aortic valve endocarditis due to Erysipelothrix rhusiopathiae in a homeless man. Case Rep Infect Dis 2013: 923034 . 